(C) 2017 IEEE. Personal use of this material is permitted. Permission from IEEE must be obtained for all other uses, in any current or future media, including reprinting/republishing this material for advertising or promotional purposes, creating new collective works, for resale or redistribution to servers or lists, or reuse of any copyrighted component of this work in other works. 


\title{
Selective Harmonic Elimination Model Predictive Control for Multilevel Power Converters
}

\author{
Ricardo P. Aguilera, Member, IEEE, Pablo Acuna, Member, IEEE, Pablo Lezana, Member, IEEE, \\ Georgios Konstantinou, Member, IEEE, Bin Wu, Fellow, IEEE, Steffen Bernet, Member, IEEE, \\ and Vassilios G. Agelidis Fellow, IEEE
}

\begin{abstract}
In this work, a Model Predictive Control (MPC) strategy that combines Finite-Control-Set MPC (FCS-MPC) with Selective Harmonic Elimination (SHE) modulation pattern in its formulation is proposed to govern multilevel power converters. Based on a desired operating point for the system state (converter current reference), an associated predefined SHE voltage pattern is obtained as a required steady-state control input reference. Then, the cost function is formulated with the inclusion of both system state and control input references. According with the proposed reference and cost function formulation, the predictive controller prefers to track the converter output current reference in transients, while preserving the SHE voltage pattern in steadystate. Hence, as evidenced by experimental results, a fast dynamic response is obtained throughout transients while a predefined voltage and current spectrum with low switching frequency is achieved in steady-state.
\end{abstract}

Index Terms-Finite control set, predictive control, pulsewidth modulation, selective harmonic elimination (SHE), multilevel converters, controller performance.

\section{INTRODUCTION}

Multilevel converters (MCs) are an interesting technology for medium/high-voltage and high power applications, such as high-power ac motor drives, active power filters, and integration of renewable energy sources to the grid [1]. MCs enable power converters to reach high power levels using mediumvoltage devices, improve the quality of output waveforms, and reduce detrimental effects, like common-mode voltage [2].

Since MCs are comprised of several internal power switch arrays, a variety of modulation techniques have been proposed to handle them [3]-[5]. They allow MCs to generate staircase switched waveforms to synthesize a desired converter output voltage with an adjustable amplitude, frequency, and

Manuscript received March 18, 2016; accepted April 27, 2014. The authors acknowledge the support of the Chilean Research Council (CONICYT) under grants FONDECYT 1140578 and Basal Project FB0008: Advanced Center for Electrical and Electronics Engineering (AC3E).

R. P. Aguilera, P. Acuna, G. Konstantinou, and V. G. Agelidis are with the Australian Energy Research Institute and the School of Electrical Engineering and Telecommunications, The University of New South Wales, Sydney, NSW 2052, Australia (e-mails: raguilera@ieee.org; pablo.acuna@unsw.edu.au; g.konstantinou@unsw.edu.au; vassilios.agelidis@unsw.edu.au).

P. Lezana is with the Department of Electrical Engineering, Universidad Técnica Federico Santa María, Valparaíso, Chile (e-mail: plablo.lezana@usm.cl).

B. Wu is with the Department of Electrical and Computer Engineering, Ryerson University, Canada (e-mail: bwu@ee.ryerson.ca).

S. Bernet is with the Professur Leistungselektronik, Elektrotechnisches Institut, Fakultät Elektrotechnik und Informationstechnik, Technische Universität Dresden, Dresden 01069, Germany (e-mail: steffen.bernet@tudresden.de) phase fundamental component that is, in general, sinusoidal in steady-state. Hence, a modulation stage for high-power converters is aimed to generate high power quality (low harmonic distortion) with minimum switching frequency (reduced commutation losses). These two requirements compete with each other and, therefore, it is considered one of the major challenges in high power MCs [1]. One of the earliest modulation technique to address this issue is Selective Harmonic Elimination PWM (SHE-PWM) [6]. This multilevel modulation technique provides a desired converter output voltage spectrum of a PWM power converter by optimally eliminating selected, typically low-order, harmonics [6]. To do this, a Fourier decomposition over one fundamental period of a PWM voltage waveform is performed. Thus, the transition instants (or switching angles $\alpha_{i}$ ) are optimally obtained in order to eliminate some undesired harmonics while regulating the fundamental component to the required value. As a result, high quality waveforms with low switching to fundamental frequency ratio and low switching losses are obtained [7]. Despite these favorable features, implementing a closed-loop control strategy for power converters when using SHE-PWM is not a trivial task [8].

Regarding the control of power converters, Model Predictive Control (MPC) has emerged as a promising alternative to govern MCs [9]. MPC obtains the control input to be applied to the system by solving, at each sampling instant, an optimization problem which forecasts the future system behavior over a finite horizon. Several predictive control embodiments have been proposed in the literature to govern power converters, showing that these approaches may, in some cases, outperform traditional PWM-based controllers. Among them, Finite-Control-Set MPC (FCS-MPC) is one of the most popular predictive controller for power converters due to its flexibility and potentiality [10]-[13]. FCS-MPC expressly considers the power switches (or voltage levels) in the optimization problem as constraints on the control inputs [14]. To solve the optimization problem, all the possible switching combinations are evaluated in the cost function. Then, the optimal power switch combination, which minimizes the cost function, is directly implemented as a control input of the power converter. Thus, modulation stages to handle power switches are not required.

Despite the advantages that FCS-MPC offers in principle, there are some issues that still remain open. One of these problems is related to the steady-state error obtained when using FCS-MPC. To alleviate this issue, modifications to the 
cost function have been proposed [15]. On the other hand, it is well known that FCS-MPC leads to spread voltage and current spectra [11]. To deal with this disadvantage, an FCSMPC strategy that can shape the load current spectrum was proposed in [16]. Here, an apparent switching frequency is obtained by filtering the converter output current. Nevertheless, the resulting switch commutations remain unknown. Then in [17], a dual-stage predictive controller was proposed to achieve a constant switching frequency. Here, FCS-MPC is used to quickly lead the system state closed to the reference. Then, a modulated Explicit MPC is applied to finally achieve the desired system reference. Thus, a fast dynamic response is obtained during transients while a fixed switching frequency and commutations are achieved in the steady-state. Even though combining two controllers might offer some advantages, this solution has been only proposed for high frequency PWM converters.

Recently, an MPC method that generates an SHE-like pattern has been proposed in [18]. This approach is based on a sliding discrete Fourier transform (SDFT) that calculates voltage harmonics in real-time. Thus, the MPC strategy uses this information to mitigate undesired harmonics. However, SDFTs require, in general, a large sampling window (at least one fundamental period) to properly calculate these harmonics. Therefore, the resulting closed-loop dynamic is forced to be slow.

In the work at hand, an MPC strategy that combines FCS-MPC with SHE-PWM in its formulation is proposed to govern MCs. The key novelty of this proposal is to define a desired steady-state operation point of the converter in terms of currents and voltages. Thus, based on this desired system state (converter current) reference, an associated pre-calculated SHE voltage pattern is considered as the required steady-state control input reference. Then, both the system state and the control reference are included into the cost function. As a result, the proposed predictive controller prefers to track the current reference during transients while preserving the SHEPWM voltage pattern in steady-state. Hence, a fast dynamic response is obtained throughout transients while a predefined voltage and current spectrum with low commutation frequency is achieved in steady-state. This paper extends the preliminary work [19] by given a proper cost function design. Moreover, the quantization effect of the proposed predictive controller over the optimal SHE-PWM pattern is analyzed. Finally, experimental results of the proposed SHE-MPC strategy when governing a three-phase three-level HB converter are provided to validate the effectiveness of this proposal.

\section{Selective Harmonic Elimination PWM}

In its simplest form, the solutions of the SHE-PWM mathematical optimization problem can be found assuming quarterwave (QW) symmetry of the output voltage [7]. Under this assumption, the dc component and even harmonics of the waveform are equal to zero and consequently not considered in the definition of the SHE-PWM problem. The normalized Fourier coefficients of the $n$-th harmonic component for a three-level voltage waveform $v_{\mathrm{SHE}}$ is given in general form

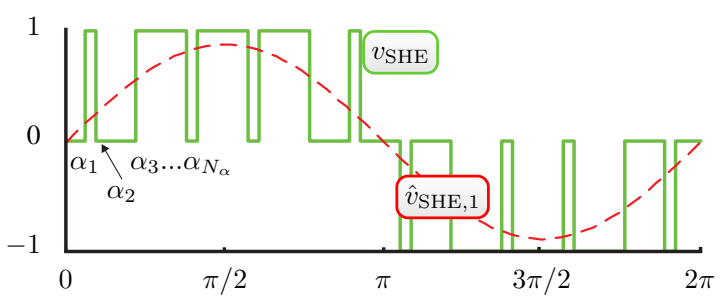

Fig. 1. Normalized three-level SHE-PWM waveform.

by

$$
b_{n}=\sum_{i=1}^{N_{\alpha}}(-1)^{i-1} \cos n\left(\alpha_{i}\right),
$$

where $n$ is the order of the harmonic, $N_{\alpha}$ is the number of switching angles $\left(\alpha_{i}\right)$ in the QW of the PWM waveform and $i \in\left\{1, N_{\alpha}\right\}$ is the order of each switching angle, as depicted in Fig. 1. The system of equations includes control of the fundamental frequency component to the required level, as defined by the modulation index $M$, and elimination of $N_{\alpha}-1$ low-order, non-triplen harmonics so that:

$$
\begin{aligned}
M & =\sum_{i=1}^{N_{\alpha}}(-1)^{i-1} \cos \left(\alpha_{i}\right), \text { and } \\
0 & =\sum_{i=1}^{N_{\alpha}}(-1)^{i-1} \cos n\left(\alpha_{i}\right),
\end{aligned}
$$

where $n \in\left\{5,7,11, \ldots, 3 N_{\alpha}-2\right\}$ for odd $N_{\alpha}$ and $n \in$ $\left\{5,7,11, \ldots, 3 N_{\alpha}-1\right\}$ for even $N_{\alpha}$. Thus, the amplitude of the fundamental frequency component $\hat{v}_{\mathrm{SHE}, 1}$ is given by:

$$
\hat{v}_{\mathrm{SHE}, 1}=\frac{4 M}{\pi},
$$

where $M \in(0,0.91)$ stands for the modulation index.

The trigonometrical system of equations defined by (2) and (3) mainly exhibits multiple solutions [20]. Therefore, a large number of methods have been proposed for the calculation of sets of solutions over the range of modulation indices, $M$. Due to the complexity of calculations involved these are typically performed off-line and include numerical iterations, optimization techniques, genetic algorithms (GAs) and the theory of resultants [7]. In this work, the solutions are acquired by minimizing a cost function defined as:

$$
F\left(\alpha_{1}, \ldots, \alpha_{i}\right)=\left(b_{1}-M\right)^{2}+b_{5}^{2}+b_{7}^{2}+\ldots+b_{3 N_{\alpha}-2}^{2},
$$

where the SHE-PWM solutions provide a cost of zero, with the constraint of

$$
0<\alpha_{1}<\alpha_{2}<\ldots<\alpha_{N_{\alpha}}<\frac{\pi}{2} .
$$

The steps for formulating the SHE-PWM problem are similar when the method is applied to single-phase converters. The major differences between the two are that triplen harmonics need to be eliminated and the operation cannot be extended to the over-modulation region $(M>\pi / 4)$. Due to the inherent advantages of three-phase SHE-PWM, the rest of the paper will focus on three-phase converters but all the following steps are equally applicable to single-phase converters. Figure 2(a) shows one set of solutions for the three-level waveform with 

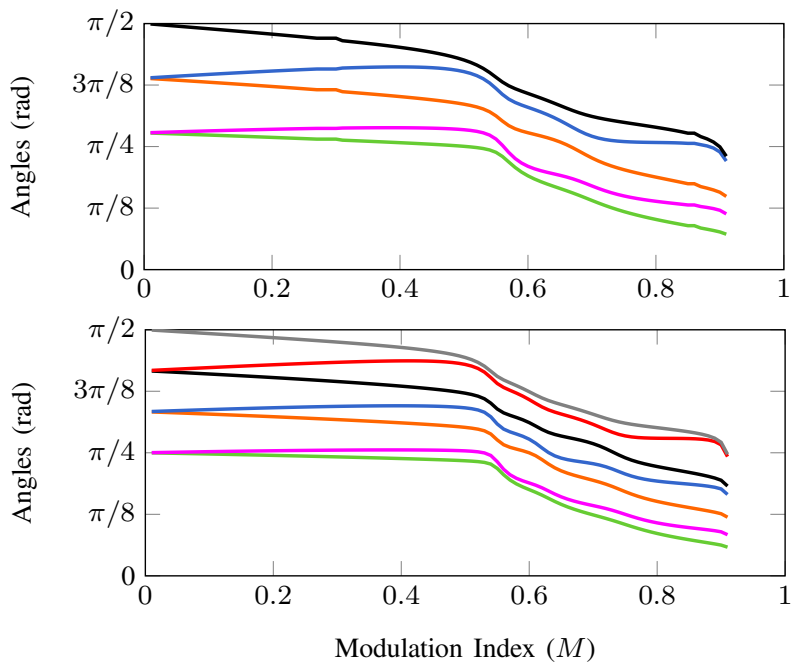

Fig. 2. Three-level SHE-PWM set of solutions with (a) five; (b) seven switching angles per quarter-period, $0<M<0.91$.

$N_{\alpha}=5$ angles and Fig. 2(b) shows one set of solutions with $N_{\alpha}=7$ angles, which eliminates the first four and six odd, non-triplen harmonics respectively.

Notice that, for three-level waveforms, multiple sets of solutions can be calculated in the higher modulation index range $(M>0.5)$ but the unique continuous solution facilitates SHE-PWM closed-loop implementation over the complete operating range and is, hence, considered in this work. As a result, solutions that are acquired through the formulation of (5) provide elimination of the low order harmonics from the converter output voltage spectrum. It is important to notice that alternative approaches vary the optimization goal to achieve targets other than complete elimination of the harmonics. Examples of such methods include i) the minimization of the waveform THD or current harmonics (also known as Optimized Pulse Patterns, OPP [8]) and ii) the restriction of each individual harmonic to limits set by grid codes (Selective Harmonic Mitigation, SHM [21]).

\section{FROM STANDARD FCS-MPC TO SHE-MPC}

This section describes how the standard FCS-MPC strategy can be enhanced to achieve an SHE-PWM pattern without combining control strategies or using real-time Fourier transformations.

Firstly, it is considered that the power converter to be controlled can be modeled, in a discrete-time state space framework, via:

$$
x(k+1)=A x(k)+B u(k),
$$

where $x(k) \in \mathbb{R}^{n}$ stands for the $n$-system states (e.g. currents and voltages) at each instant $k$ and $u(k) \in \mathbb{U} \subset R^{m}$ represents the $m$-control inputs of the power converter, i.e., the switch positions or voltage levels. Therefore, this kind of input belongs to an FCS of $p$ elements, represented by

$$
u(k) \in \mathbb{U}=\left\{u_{1}, u_{2}, \ldots, u_{p}\right\} .
$$

The control target is to track a desired converter reference, normally a sinusoidal waveform (e.g. output current), represented by $x^{\star}(k) \in \mathbb{R}^{n}$, i.e., $x(k) \rightarrow x^{\star}(k)$. A standard predictive control approach to govern this class of power converters is FCS-MPC [12].

\section{A. Standard FCS-MPC}

Standard FCS-MPC strategy operates in discrete time with sampling frequency $f_{s}=T_{s}^{-1}$ and one-step prediction horizon. Thus, at each sampling time $k$, a measurement of the system state $x(k)$ is taken and, then, a cost function is evaluated for each tentative control input element, $u^{\prime}(k)$, in $\mathbb{U}$. Generally in power electronics, the cost function only considers a positive sum of the tracking errors for each controlled variable [12]. Thus, the standard horizon-one cost function can be expressed as:

$$
J_{s t d}(k)=\left\|x^{\prime}(k+1)-x^{\star}(k+1)\right\|_{2}^{2},
$$

where $x^{\prime}(k+1)$ stands for the state predictions, generated by $u^{\prime}(k)$, based on the power converter model (7). $\|\cdot\|_{2}^{2}$ represents the quadratic Euclidean norm, i.e., $\left\|a-a^{\star}\right\|_{2}^{2}=\left(a_{1}-a_{1}^{\star}\right)^{2}+$ $\ldots+\left(a_{n}-a_{n}^{\star}\right)^{2}$, for a pair of vectors $a, a^{\star} \in \mathbb{R}^{n}$. Therefore, the optimal control input which minimizes the cost function (9) is then applied to the converter. Examples of FCS-MPC applied to power converters can be found in [10]-[13], [22][31].

\section{B. Cost Function Formulation}

Considering only the tracking error of the controlled variables in the cost function may lead, in general, to a poor closed-loop performance when controlling power converters [15]. Besides a fast dynamic response, it is also desired to obtain a reduced number of switch commutations and a predefined and fixed frequency spectrum. To achieve these inherent power electronics goals when using FCS-MPC, extra terms are normally added to the cost function; see e.g., [32].

Recently, an FCS-MPC design to govern power converters with guaranteed performance has been proposed in [33]. Here, the use of the input tracking error has been considered:

$$
J(k)=\left\|x^{\prime}(k+1)-x^{\star}(k+1)\right\|_{2}^{2}+\sigma\left\|u^{\prime}(k)-u^{\star}(k)\right\|_{2}^{2},
$$

where $u^{\prime}(k) \in \mathbb{U}$ is the tentative input combination that generates the predictions $x^{\prime}(k+1)$, and $u^{\star}(k)$ is the required input to keep the state reference, $x^{\star}(k)$, in steady-state. Here, $\sigma$ is a weighting factor that allows one to adjust a desired closed-loop performance. For faster dynamic, a smaller value for $\sigma$ must be chosen; see [33]. Notice that when the system state is near its reference, $x(k) \approx x^{\star}(k)$, the first part of the cost function tends to zero. Thus, the second term becomes the dominant term. This leads to an optimal control input, $u(k)$, that tracks the input reference, $u^{\star}(k)$, during the steadystate. Moreover, by using the cost function (10), stability and performance of the predictive closed-loop can be guaranteed [33]-[35]. 


\section{Steady-State Reference Design}

The proposed predictive controller is aimed to govern a power converter, that is mathematically described as per (7), to track the following three-phase sinusoidal output current reference:

$$
i_{y}^{\star}(t)=I^{\star} \sin \left(\omega t+\phi_{y}\right),
$$

where $I^{\star}$ represents the output current reference amplitude for all $y \in\{a, b, c\}$. Moreover, $\phi_{a}=0, \phi_{b}=-2 \pi / 3$, and $\phi_{c}=2 \pi / 3$.

To keep the desired current reference (11), the power converter must apply the following per-unit voltage in the steady-state:

$$
m_{y}^{\star}(t)=m^{\star} \sin \left(\omega t+\phi_{y}+\delta^{\star}\right) .
$$

Notice that $m_{y}^{\star}(t)$ in (12) is a continuous (in magnitude) variable, i.e., $m_{y}^{\star}(t) \in \mathbb{R}$. As shown in [33], using $m_{y}^{\star}(t)$ as an input reference in (10) may lead to a high switching frequency. Therefore, this work proposes to use its associated optimal SHE-PWM pattern $v_{S H E}^{\star}(t)$, that generates $m_{y}^{\star}(t)$ as its fundamental component, as control input reference, i.e., $u^{\star}(k)=v_{S H E}^{\star}(k)$.

Then, considering that the optimal angles presented in Fig. 2 are stored in a look-up table, it is necessary to determine the modulation index $m^{\star}$ and the angle $\delta^{\star}$ between the current reference and the required converter output voltage. Using these two parameters, one can read the SHE-table to obtain the associated SHE voltage reference, $v_{\mathrm{SHE}}^{\star}(t)$, which is optimally obtained as described in Section II. This is expressed via:

$$
v_{\mathrm{SHE}}^{\star}(t)=\operatorname{SHE}\left(m^{\star}, \delta^{\star}\right)=\left[\begin{array}{c}
v_{\mathrm{SHE}, \mathrm{a}}^{\star}(t) \\
v_{\mathrm{SHE}, \mathrm{b}}^{\star}(t) \\
v_{\mathrm{SHE}, \mathrm{c}}^{\star}(t)
\end{array}\right] .
$$

\section{Proposed SHE-MPC}

Finally, to achieve the desired three-phase sinusoidal currents with a predefined spectrum, the proposed SHE-MPC is implemented in discrete-time by using the cost function, $J(k)$ as per (10), with $x^{\star}(k)$ and $u^{\star}(k)$ as the sampled value of (11) and (13) respectively, i.e.,

$$
J(k)=\left\|i_{a b}^{\prime}(k+1)-i_{a b}^{\star}(k+1)\right\|_{2}^{2}+\sigma(k)\left\|v_{a b c}^{\prime}(k)-v_{S H E}^{\star}(k)\right\|_{2}^{2},
$$

where $v_{a b c}(k)$ stands for the converter output voltage levels. Thus, $v_{a b c}^{\prime}(k)$ is the tentative control input voltage vector that leads to the current predictions $i_{a b}^{\prime}(k+1)$. This relationship is given by the discrete-time converter model

$$
i_{a b}^{\prime}(k+1)=A i_{a b}(k)+B v_{a b c}^{\prime}(k) .
$$

As previously mentioned, the weighting factor $\sigma(k)$ is used to adjust the closed-loop performance of the proposed predictive controller [33]. Thus, when a small value is chosen, i.e., $\sigma(k) \approx 0, J(k)$ in (14) will tend to the standard cost function $J_{s t d}(k)$ in (9). This will enforce a fast dynamic response when a transient is detected while producing large commutations in the steady-state. On the other hand, for a large value, i.e., $\sigma(k) \gg 0$, a slow dynamic response will be obtained. However, an input close or equal to the control

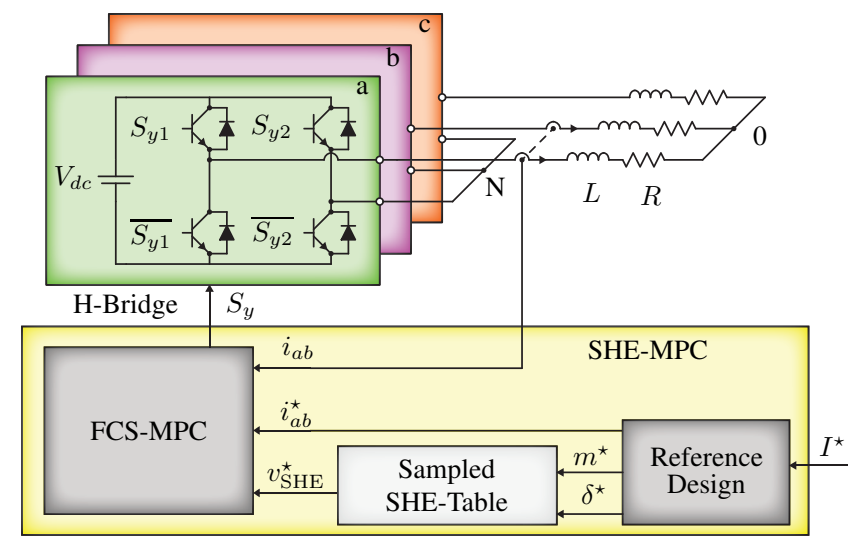

Fig. 3. Three-phase three-level HB schematic and block diagram of the proposed SHE-MPC.

reference, i.e., $v_{a b c}^{\prime}(k) \approx v_{\mathrm{SHE}}^{\star}(k)$, will be generated in the steady-state, which will help to promote an SHE-PWM pattern on the converter output voltage. Motivated by this, the use of a variable weighting factor $\sigma(k)$ is proposed in this work. Firstly, a normalized system state deviation is introduced

$$
\Delta i(k)=\frac{\left(i_{a b}(k)-i_{a b}^{\star}(k)\right)^{T}\left(i_{a b}(k)-i_{a b}^{\star}(k)\right)}{I_{\max }^{\star}} \geq 0,
$$

where $I_{\max }^{\star}$ is the maximum amplitude of the current reference. Thus, the variable weighting factor is chosen as

$$
\bar{\sigma}(k)=\sigma_{\max }-\lambda \Delta i(k) .
$$

This, after including saturations, becomes

$$
\sigma(k)= \begin{cases}\bar{\sigma}(k) & \text { if } \sigma_{\min } \leq \bar{\sigma}(k) \leq \sigma_{\max } \\ \sigma_{\min } & \text { if } \bar{\sigma}(k)<\sigma_{\min }\end{cases}
$$

where $0 \leq \sigma_{\min }<\sigma_{\max }$. Here, $\sigma_{\min }$ allows one to adjust the closed-loop dynamic response of the controller while $\sigma_{\max }$ determines how closely the resulting optimal control input can resemble the optimal SHE-PWM pattern during the steadystate.

It is important to emphasize that, since $v_{a b c}(k)$ is chosen to be the output voltage level, both $v_{a b c}^{\prime}(k)$ and $v_{\mathrm{SHE}}^{\star}(k)$ in (10) belong to the same FCS $\mathbb{U}^{3}$. This makes the SHEPWM reference (13) compatible with the actual system input. Nonetheless, since the proposed FCS-MPC works in discretetime, $v_{S H E}(k)$ is a sampled-version of the optimal $v_{S H E}(t)$. Therefore, proper cancellation of undesired harmonics could be compromised. Effects of the sampling over the resulting SHE-PWM pattern are studied in Section V-A.

\section{CASE Study: Three-Level HB CONVERTER}

To highlight its benefits, in this section the proposed SHEMPC is used to govern a three-phase three-level HB converter, where each $\mathrm{HB}$ cell is electrically fed with isolated dc-voltage sources as shown in Fig. 3. For the sake of the clarity, only a passive RL-load is considered as in [18]. Further cases, such as grid-connected converters and electrical drives will be studied in a future work. 


\section{A. Continuous-Time Model}

Considering the three-level HB converter depicted in Fig. 3, by using simple circuit analysis, the following continuous-time dynamic model for each output current can be obtained:

$$
\begin{aligned}
\frac{d i_{y}(t)}{d t} & =-\frac{R}{L} i_{y}(t)+\frac{1}{L}\left(v_{y N}(t)-v_{0 N}(t)\right), \\
v_{y N}(t) & =V_{d c} v_{\ell y}(t),
\end{aligned}
$$

where $v_{y N}(t)$ stands for the total converter output voltage per phase, and

$$
v_{\ell y}(t) \in \mathbb{V}=\{-1,0,1\}
$$

is the vector that represents the levels of the converter output voltage. Additionally, $v_{0 N}(t)$ is the, so-called, common-mode voltage, which is given by

$$
v_{0 N}(t)=\frac{1}{3}\left(v_{a N}(t)+v_{b N}(t)+v_{c N}(t)\right) .
$$

\section{B. Discrete-Time Model}

In this work, the proposed SHE-MPC for a three-phase three-level $\mathrm{HB}$ converter is formulated in the original $a b c$ framework. Moreover, the use of the phase voltage levels as control input is considered. Thus, only 27 input (voltage level) combinations needs to be evaluated (instead of 64 when considering power switches). Therefore, the system state and control input are chosen as

$$
i_{a b}(k)=\left[\begin{array}{l}
i_{a}(k) \\
i_{b}(k)
\end{array}\right] \in \mathbb{R}^{2}, \quad v_{a b c}(k)=\left[\begin{array}{l}
v_{\ell a}(k) \\
v_{\ell b}(k) \\
v_{\ell c}(k)
\end{array}\right] \in \mathbb{U},
$$

where $i_{c}(k)=-\left(i_{a}(k)+i_{b}(k)\right)$ and the input, $v_{a b c}(k)$, belongs to the following FCS:

$$
\mathbb{U}=\mathbb{V}^{3}=\{-1,0,1\}^{3} .
$$

Therefore, by applying a simple forward Euler discretization to (19) with a sampling period of $T_{s}$, the HB converter adopts the discrete-time model in (15) with

$$
\begin{aligned}
A & =\left[\begin{array}{cc}
1-\frac{R T_{s}}{L} & 0 \\
0 & 1-\frac{R T_{s}}{L}
\end{array}\right], \\
B & =\frac{V_{d c} T_{s}}{3 L}\left[\begin{array}{ccc}
2 & -1 & -1 \\
-1 & 2 & -1
\end{array}\right] .
\end{aligned}
$$

A block diagram of the proposed SHE-MPC strategy is also included in Fig. 3. Inside this block, the reference design block receives the current reference $I^{\star}$ which is limited by:

$$
I_{\max }^{\star}=\hat{v}_{\max } / Z
$$

where, from $(4), \hat{v}_{\max }=\left(4 \times 0.91 V_{d c}\right) / \pi$ and $Z=$ $\sqrt{R^{2}+X_{L}^{2}}$ with $X_{L}=\omega L$.

For the three-phase three-level converter shown in Fig. 3, the modulation index and the angle between the applied voltage and current are defined as:

$$
\begin{aligned}
m^{\star} & =\frac{\pi}{4} \frac{Z\left|I^{\star}\right|}{V_{d c}} \\
\delta^{\star} & =\tan ^{-1}\left(\frac{X_{L}}{R}\right) .
\end{aligned}
$$

After that, the function $\operatorname{SHE}(\cdot, \cdot)$ reads the look-up table and gives $v_{\mathrm{SHE}}^{\star}$, to keep the desired current reference (11) in the steady-state. Consequently, the optimal converter output voltage level given by the proposed SHE-MPC is the one that minimizes the cost function (14), i.e.,

$$
v_{a b c}^{o p}(k)=\arg \left\{\min _{v_{a b c} \in \mathbb{U}} J(k)\right\},
$$

which is obtained by evaluating all the possible voltage level combinations $\left(v_{a b c} \in \mathbb{U}\right.$ as per (23)) in $J(k)$. Then, a Sorting Algorithm (SA) to spread the power losses among the power switches is used to finally implement the optimal converter output voltage level, i.e.,

$$
S_{a b c}^{o p}(k)=\operatorname{SA}\left\{v_{a b c}^{o p}(k)\right\} .
$$

\section{Results}

In this section, numerical and experimental results are provided to adjust and validate the effectiveness and performance of the proposed SHE-MPC when governing a three-phase three-level HB converter.

\section{A. Quantization effect over the optimal SHE-PWM pattern}

When solving the optimization problem presented in Section II, for a given modulation index $M$, a set of $N$-optimal continuous-valued angles, $\alpha_{j} \in(0, \pi / 2)$, is obtained. Thus, it is possible to generate, for one fundamental period, a continuous SHE-PWM pattern $v_{S H E}(t)$. Unlike SHE-PWM implementations that store the angles in a look-up table, the proposed SHE-MPC requires one to store a sampled-version of this pattern, $v_{S H E}(k)$. To do this, the same sampling frequency, $f_{s}$, used by the FCS-MPC is considered. Thus, the sampled-version of the $N$-optimal angles, $\hat{\alpha}_{j}$, is constrained to belong to the following finite set:

$$
\hat{\alpha}_{j} \in\left\{0, \Delta_{\delta}, 2 \Delta_{\delta}, \ldots, \pi / 2\right\},
$$

where

$$
\Delta_{\delta}=\frac{2 \pi}{N_{s}}, \quad N_{s}=\frac{f_{s}}{f_{o}} .
$$

Here, $N_{s}$ stands for the number of samples within a fundamental period $T=1 / f_{o}$.

Therefore, the sampling process introduces a quantization effect on the optimal angles which can be represented by

$$
\hat{\alpha}_{j}=q\left\{\alpha_{j}\right\}=\alpha_{j}+\eta\left(f_{s}\right),
$$

where $\eta\left(f_{s}\right)$ is the resulting quantization noise that depends on the sampling frequency, $f_{s}$, and is bounded by:

$$
\left|\eta\left(f_{s}\right)\right| \leq \Delta_{\delta} / 2 .
$$

Then, to read the lookup table to obtain the voltage reference, $v_{S H E}^{\star}(k)$, the voltage reference angle, $\delta^{\star}$, is restricted to belong to the following finite set:

$$
\delta^{\star} \in\left\{0, \Delta_{\delta}, 2 \Delta_{\delta}, \ldots, 2 \pi\right\} .
$$

As a consequence of this quantization, the elimination of undesired harmonics may be compromised since the quantized angles may differ from the optimal ones, i.e., $\hat{\alpha}_{j} \neq \alpha_{j}$. This quantization effect is depicted in Fig. 4 for the case of $N_{\alpha}=5$ 
(a) 8

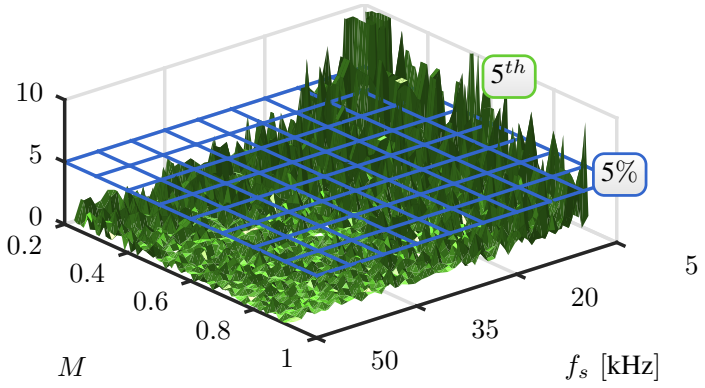

(b)

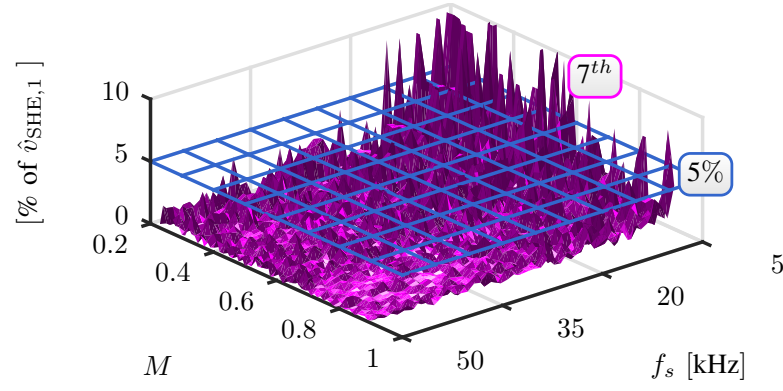

(c)

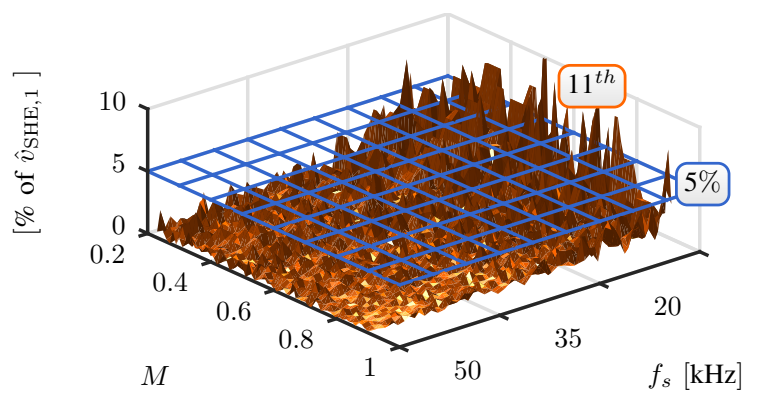

(d)

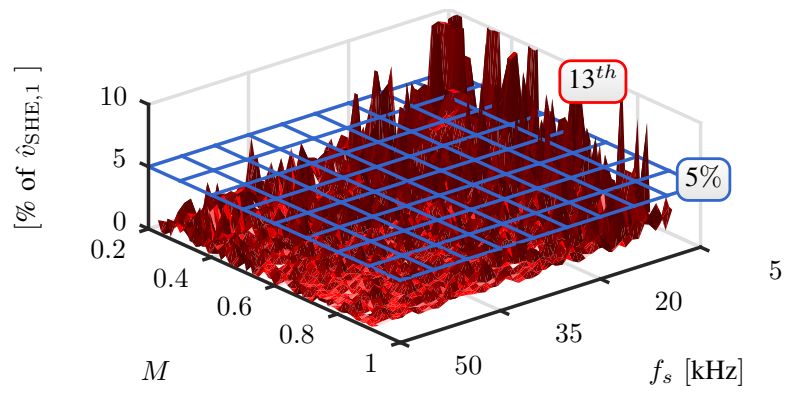

Fig. 4. Quantization effect over the SHE-PWM pattern for $N_{\alpha}=5$ and $f_{o}=50 \mathrm{~Hz}$ : (a) $5^{t h}$ harmonic elimination; (a) $7^{t h}$ harmonic elimination; (a) $11^{\text {th }}$ harmonic elimination; (a) $13^{\text {th }}$ harmonic elimination.

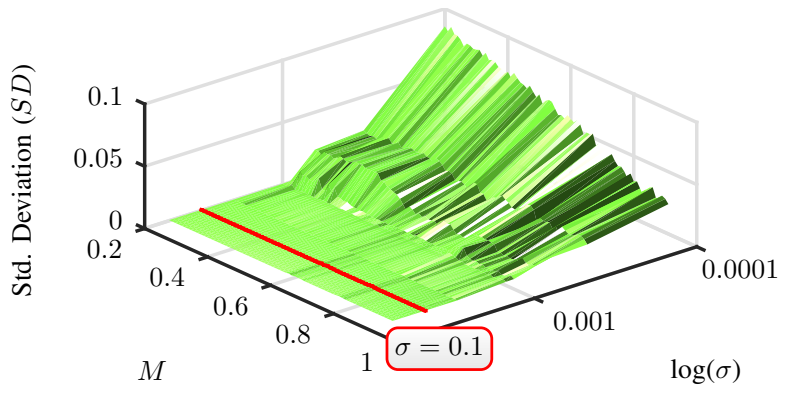

Fig. 5. Standard Deviation of $v_{S H E}$ over $v_{\mathrm{SHE}}^{\star}$ under weighting factor and modulation index variation.
Table I

SYSTEM PARAMETERS

\begin{tabular}{ccc}
\hline \hline Variable & Description & Value \\
\hline$S_{r}$ & Total rated apparent power & $4.45 \mathrm{kVA}$ \\
$V_{d c}$ & dc capacitor voltage per HB & $148 \mathrm{~V}$ \\
$L$ & Load inductor & $25 \mathrm{mH}$ \\
$r$ & Load resistance & $10 \Omega$ \\
$f_{s}$ & Sampling frequency & $20 \mathrm{kHz}$ \\
$I_{\max }^{\star}$ & Maximum Current Reference & $13.5 \mathrm{~A}$ \\
$\sigma_{\max }$ & Controller Setting & 0.1 \\
$\sigma_{\min }$ & Controller Setting & 0.001 \\
$\lambda$ & Controller Setting & 2 \\
\hline \hline
\end{tabular}

angles to eliminate the $5^{\text {th }}, 7^{\text {th }}, 11^{\text {th }}$, and $13^{\text {th }}$ harmonics with a fundamental frequency of $f_{o}=50 \mathrm{~Hz}$. Here, one can clearly see that as the sampling frequency is increased the harmonic cancellation is improved. Moreover, with a sampling frequency larger than $20 \mathrm{kHz}$, the amplitude of these four harmonics is reduced to a value smaller than $5 \%$ for $M \in(0.2,0.91)$. Consequently, the proposed SHE-MPC is implemented with $f_{s}=20 \mathrm{kHz}$, which implies a quantization error less than $0.45^{\circ}$ $\left(\Delta_{\delta}=0.9^{\circ}\right)$.

\section{B. Weighting factor selection}

One of the key features of the proposed predictive controller is related to the ability to provide a desired SHE-PWM pattern in the steady state. In this condition $\Delta i(k) \approx 0$; thus, the variable weighting factor in $(18)$ becomes $\sigma(k) \approx \sigma_{\max }$. Consequently, the controller setting that helps to provide an SHE-PWM pattern is $\sigma_{\max }$. To see the effect of $\sigma_{\max }$ over the SHE-PWM pattern, $v_{S H E}(k)$, the optimal converter voltage provided by the proposed SHE-MPC, $v_{a b c}^{o p}(k)$, during the steady-state for several values of $\sigma_{\max }$ is compared in terms of the resulting standard deviation (SD), i.e.,

$$
S D\left(\sigma_{\max }\right)=\frac{1}{N_{s}} \sqrt{\sum_{k=1}^{N_{s}}\left(v_{a b c}^{o p}(k)-v_{\mathrm{SHE}}^{\star}(k)\right)^{2}},
$$

where $N_{s}$ is as per (30). The simulation result of this analysis is presented in Fig. 5. Here, it is possible to observe that when $\sigma_{\max }<0.001$, the optimal converter voltage provided by the SHE-MPC differs from the desired SHE-PWM pattern, i.e., $v_{a b c}^{o p}(k) \neq v_{S H E}^{\star}(k)$. In fact, when $\sigma_{\max }=0$ the proposed SHE-MPC becomes the standard FCS-MPC, since $J(k)=$ $J_{s t d}(k)$; see Section III. On the other hand, $\sigma_{\max }$ cannot be too large since it can reduce the closed-loop performance; see [33]. Consequently, a value of $\sigma_{\max }=0.01$ is chosen.

During transients, i.e., $\Delta i(k) \gg 0$, the weighting factor becomes $\sigma(k) \approx \sigma_{\min }$. To ensure a fast dynamic response, a relatively small value compared to $\sigma_{\max }$ must be chosen. However, it cannot be too small to avoid obtaining a standard FCS-MPC behavior. In this case, it is chosen as $\sigma_{\min }=0.001$, 


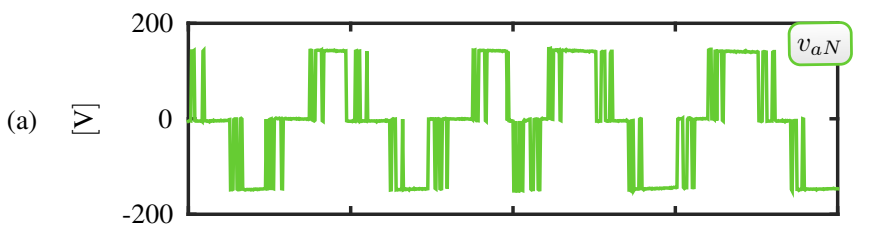

(b) $\sum 0_{-200}^{200}$
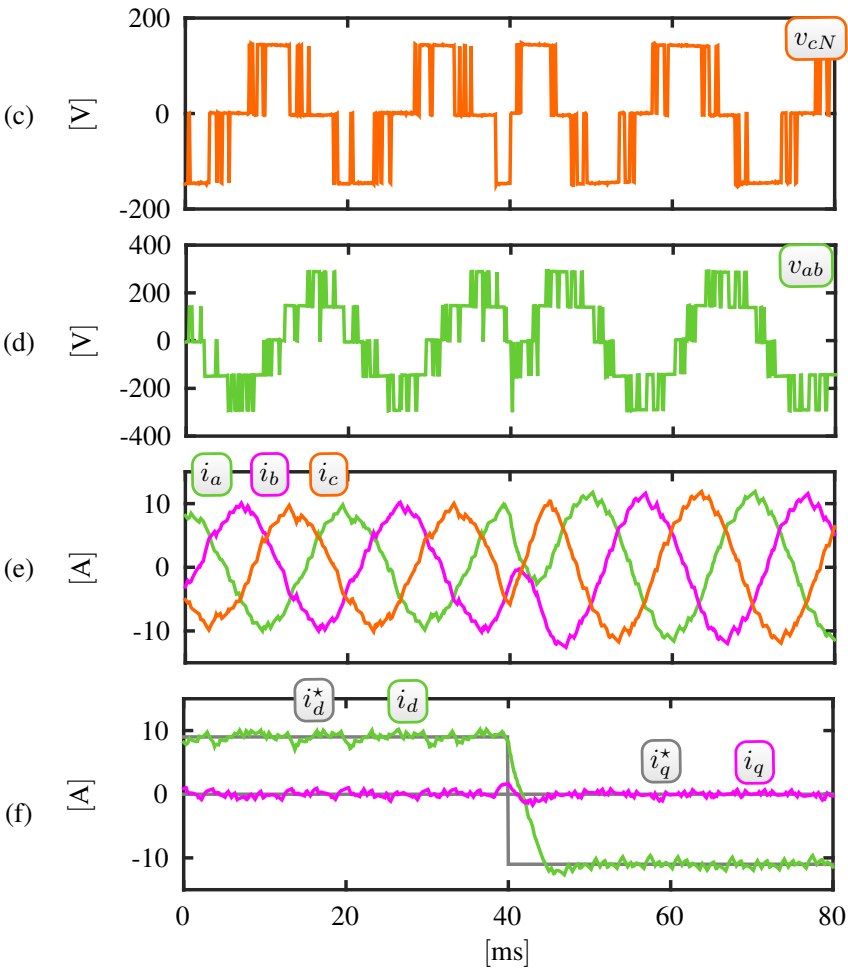

Fig. 6. PI+SHE-PWM performance with a closed-loop bandwidth of $90 \mathrm{~Hz}$ : (a) $v_{a N}(t)$; (b) $v_{b N}(t)$; (c) $v_{c N}(t)$; (d) $v_{a b}(t)$; (e) $i_{a b c}(t)$; (f) $i_{d q}(t)$.

since this value still can provided an acceptable SHE-PWM pattern, as observed in Fig. 5. It is important to note that this value is only applied for a short-time, since $\sigma(k)$ increases as the output current approaches to its reference, i.e., $\Delta i(k) \longrightarrow 0$. Finally, the controller setting $\lambda$ is tuned to obtain a smooth transition between $\sigma_{\min }$ to $\sigma_{\max }$. If this parameter is too small, i.e., $\lambda \approx 0$, then $\sigma(k) \approx \sigma_{\max }$, which will dramatically reduce the dynamic response of the controller. Conversely, if $\lambda$ is too large, then $\sigma(k) \approx \sigma_{\min }$, which will deteriorate the steady-state SHE-PWM pattern. The selection of this parameter strongly depends on the natural converter dynamics. Therefore, it has to be adjusted throughout simulations. As an initial value, it is suggested to tunned it as $\lambda=1$. Then, it can be varied to obtain a desired closed-loop dynamic performance.

\section{Experimental Validation}

The main system parameters of the experimental setup are presented in Table I. The control algorithm was implemented on a dSPACE DS1106 system, where the proposed strategy
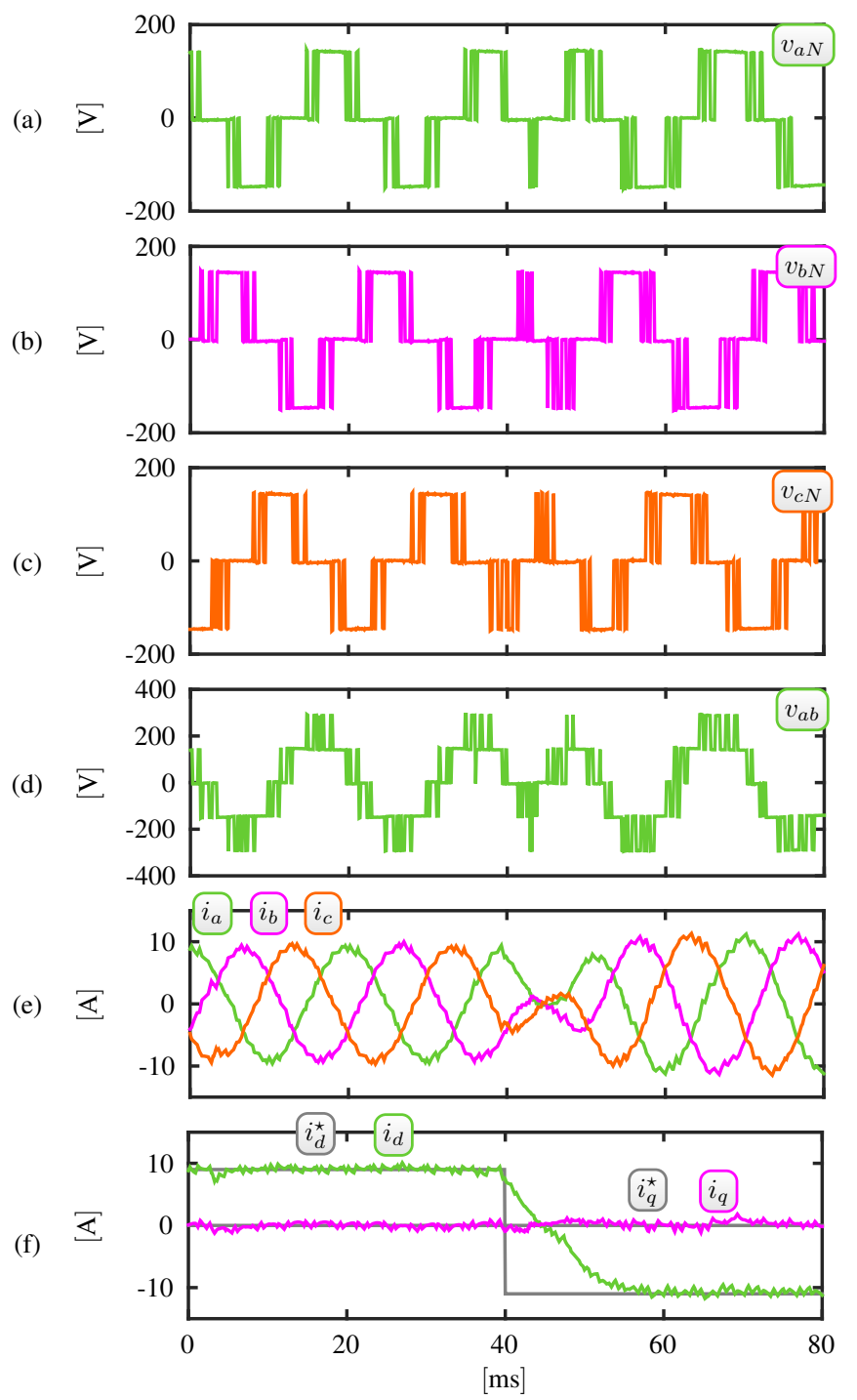

Fig. 7. PI+SHE-PWM performance with a closed-loop bandwidth of $30 \mathrm{~Hz}$ : (a) $v_{a N}(t)$; (b) $v_{b N}(t)$; (c) $v_{c N}(t)$; (d) $v_{a b}(t)$; (e) $i_{a b c}(t)$; (f) $i_{d q}(t)$.

was programmed in C. To account for the linear controllers, a PWM stage to trigger the power switches was implemented in a DS5203 FPGA board.

As an initial test, a step-change in the current reference from 9A to -11A is applied with an SHE-PWM pattern with $N_{\alpha}=5$ angles, as depicted in Fig. 2(a). Firstly, the converter is governed with a standard PI controller which generates the reference for the SHE-PWM modulator. This PI controller is initially designed to obtain a closed-loop bandwidth of $90 \mathrm{~Hz}\left(\mathrm{PI}_{90}\right)$, yielding to the converter voltages and currents depicted in Fig. 6. Additionally, a $d q$ representation of the load current is also included. Here, one can observe that the resulting currents present a non-desired steady-state oscillation. Moreover, the controller introduces more commutations than those required by the optimal SHE-PWM. To improve this, a second PI controller set with a lower closed-loop bandwidth of $30 \mathrm{~Hz}\left(\mathrm{PI}_{30}\right)$ is tested. The results are presented in Fig. 7. Here, it is possible to see that, in steady-state, the number of commutations is closer to the optimal SHE-PWM pattern when compared to the $\mathrm{PI}_{90}$ case. However, during the 


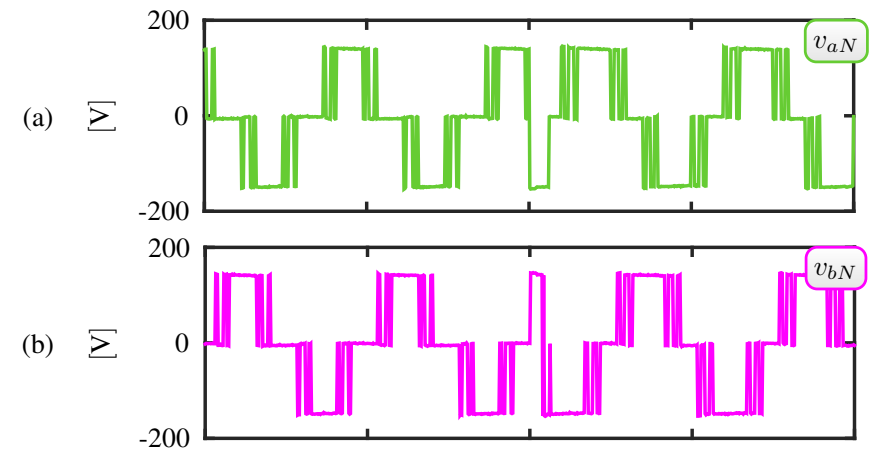

(c) $\sum \underbrace{200}_{-200}$

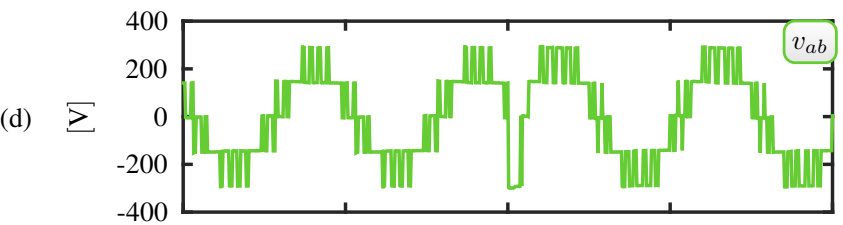

(e)

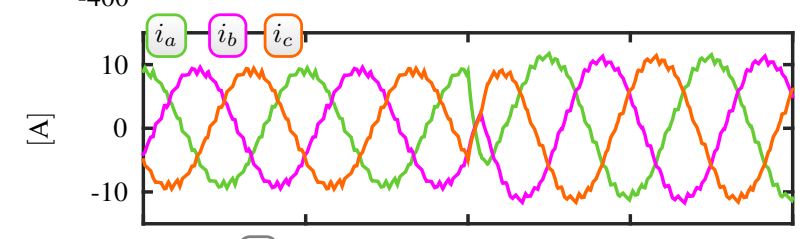

(f)

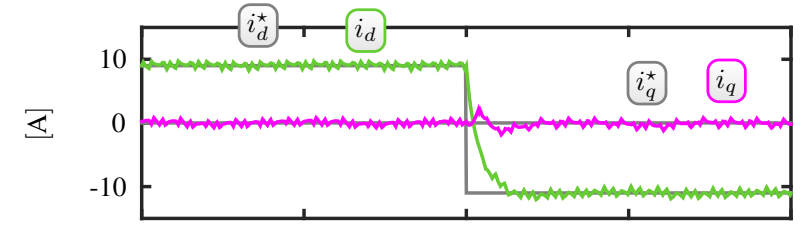

(g)

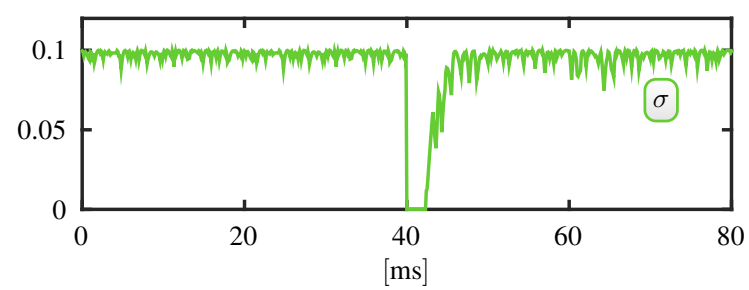

Fig. 8. Proposed SHE-MPC performance: (a) $v_{a N}(t)$; (b) $v_{b N}(t)$; (c) $v_{c N}(t)$; (d) $v_{a b}(t)$; (e) $i_{a b c}(t)$; (f) $i_{d q}(t)$; (g) $\sigma(t)$.

transient, a slower closed-loop dynamic is achieved due to the reduced controller bandwidth. Moreover, a slight steady-state oscillation can be observed.

Then, the same test is performed when the converter is governed by the proposed SHE-MPC strategy. This result is depicted in Fig. 8. For a current reference of 9A and the given load, the required steady-state modulation index and voltage angle are $m^{\star}=0.60$ and $\delta^{\star}=38^{\circ}$ respectively. As can be seen in Fig. 8(g), initially during the steady-state, the weighting factor adopts a value close to $\sigma_{\max }=0.1$. Therefore, in this situation, the steady-state SHE-PWM pattern prevails; see Fig. 5. Then, at the instant $t=40 \mathrm{~ms}$ a voltage step to $-11 \mathrm{~A}$ is introduced. This produces an increment in the converter current deviation $\Delta i(k)$. The proposed SHE-MPC responds to this variation by decreasing the weighting factor
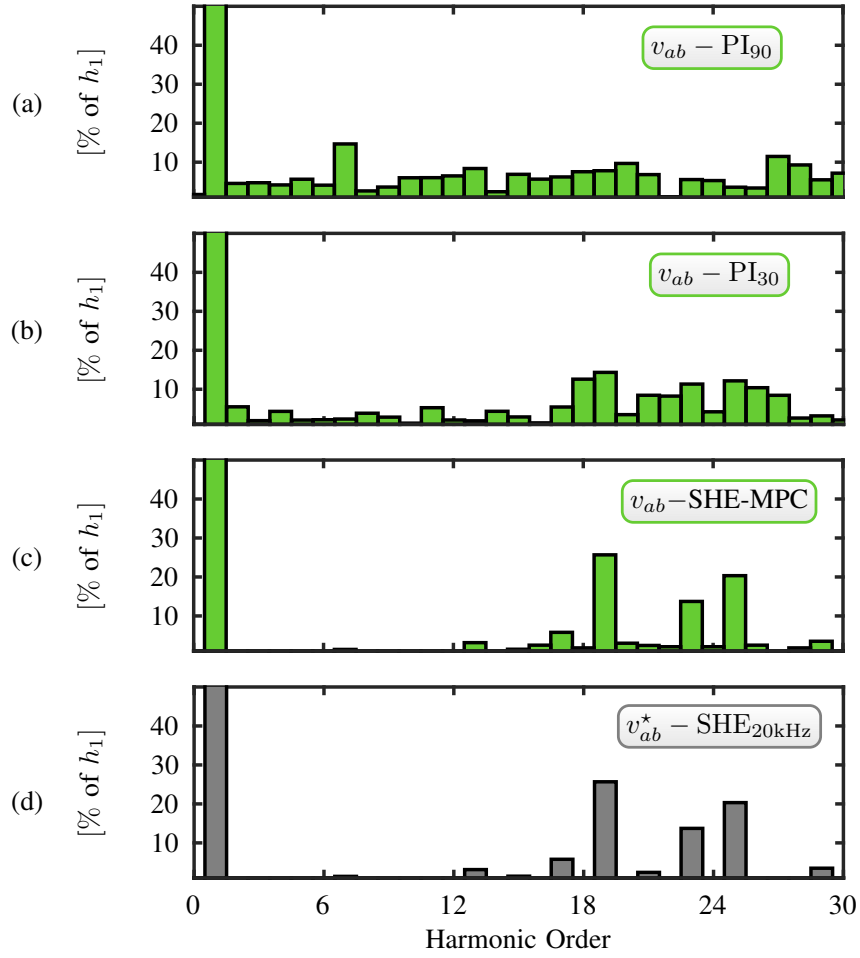

Fig. 9. Resulting line-to-line inverter output voltage spectrum for: (a) PI with a $90 \mathrm{~Hz}$ closed-loop bandwidth; (b) PI with a $30 \mathrm{~Hz}$ closed-loop bandwidth; (c) Proposed SHE-MPC; (d) sampled $v_{a b}^{\star}$ reference for $m^{\star}=0.60$.

Table II

HARMONIC ANALYSIS OF $v_{a b c}$

\begin{tabular}{|c|c|c|c|c|c|c|}
\hline \multirow{2}{*}{ Case } & \multicolumn{4}{|c|}{ Harmonic Order } & \multirow{2}{*}{$\mathrm{THD}_{v}$} & \multirow{2}{*}{$\mathrm{THD}_{i}{ }^{\mathrm{b}}$} \\
\hline & 5 & 7 & 11 & 13 & & \\
\hline $\mathrm{PI}_{90}$ & 5.62 & 14.68 & 6.02 & 8.37 & 40.02 & 7.34 \\
\hline $\mathrm{PI}_{30}$ & 2.13 & 2.36 & 5.26 & 1.90 & 41.06 & 5.57 \\
\hline SHE-MPC & 0.68 & 1.39 & 0.36 & 3.01 & 42.65 & 4.55 \\
\hline SHE-PWM Ref ${ }^{a}$ & 0.68 & 1.40 & 0.35 & 3.14 & 41.74 & - \\
\hline
\end{tabular}

$\sigma(k)$. Thus, the predictive controller modifies the SHE-PWM pattern to quickly (about $5 \mathrm{~ms}$ ) lead the current to its new reference. After this transient, the proposed SHE-MPC adopts the new optimal SHE-PWM pattern to maintain the desired 11A during the steady-state, i.e., $m^{\star}=0.78$ and $\delta^{\star}=-142^{\circ}$.

Regarding the steady-state performance, the resulting converter voltage spectra for each controller previously tested are presented in Fig. 9. Here, one can observe that the low frequency harmonic elimination target is not achieved when using conventional PI controllers. For instance, when using the $\mathrm{PI}_{30}$ controller, the $5^{\text {th }}$ harmonic presents an amplitude of $2.13 \%$ of the fundamental component. In the case of using the $\mathrm{PI}_{90}$ controller, this harmonic is even larger, 5.62\%. Clearly, the $\mathrm{PI}_{90}$ controller is trying to cancel high order harmonics due to its relatively large bandwidth. On the other hand, the $\mathrm{PI}_{30}$ controller generates a voltage spectrum closer to the optimal SHE-PWM pattern but with lower dynamic response. For the 


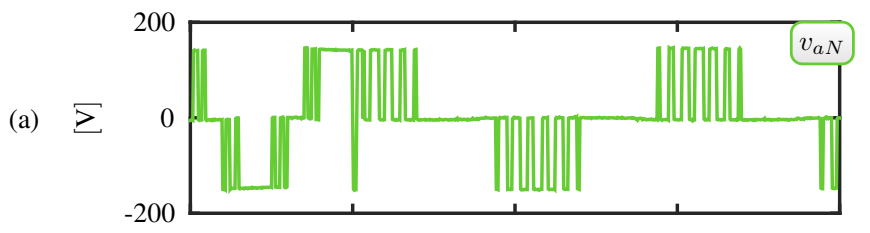

:

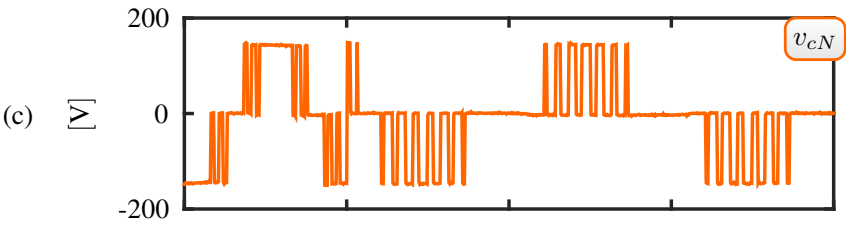

(d)

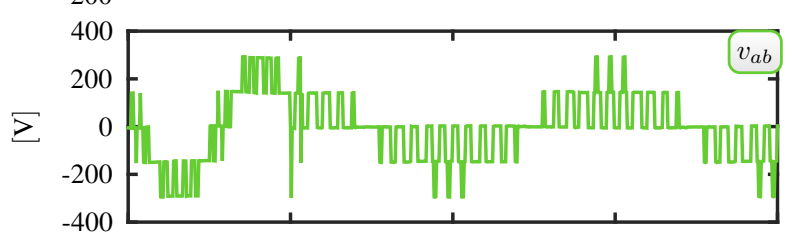

(e)

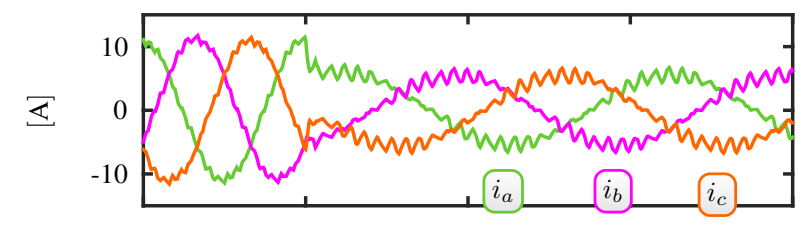

(f)

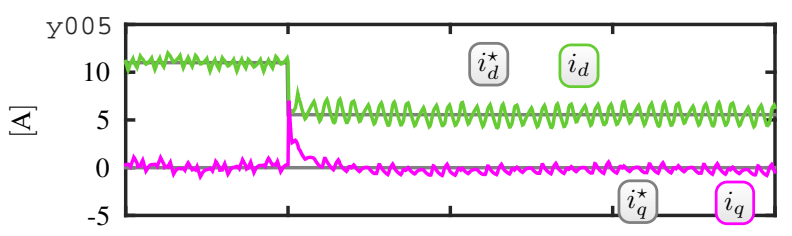

(g)

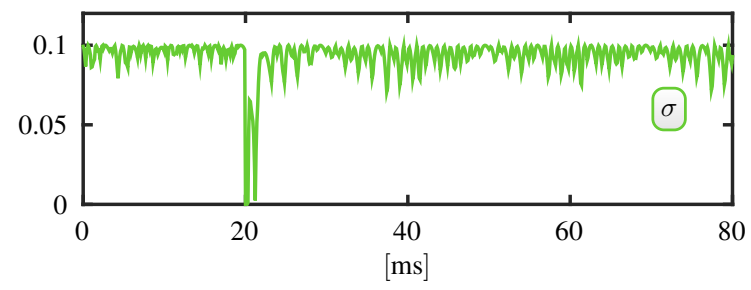

Fig. 10. Proposed SHE-MPC strategy: Amplitude/frequency step: (a) $v_{a N}(t)$; (b) $v_{b N}(t)$; (c) $v_{c N}(t)$; (d) $v_{a b}(t)$; (e) $i_{a b c}(t)$; (f) $i_{d q}(t)$; (g) $\sigma(t)$.

proposed SHE-MPC, the resulting $5^{\text {th }}$ harmonic amplitude is $0.68 \%$. The resulting low order harmonics are not exactly zero as shown in Table II since the predictive controller uses sampled version of the actual optimal SHE-PWM pattern (see Fig. 9(d)). This slightly modifies the resulting spectrum, as discussed in Section V-A. Therefore, the proposed SHE-MPC is the only controller, here analyzed, that can properly track the optimal SHE-PWM pattern with a relatively small error. Notice that the PI controllers also introduce inter-harmonics due to the current tracking oscillation, which are not observed with the proposed SHE-MPC strategy. It is also important to emphasize that the proposed predictive controller is able to achieve an optimized voltage pattern (SHE-PWM in this case) using only a horizon-one prediction. This is a major contribution since, until now, only long-horizon predictive control formulations have been shown to have the potential to achieve a performance similar to that of optimized voltage patterns [27].

To test the transient between two sets of SHE-PWM patterns, in Fig. 10 a step in both amplitude and frequency is introduced. Here, the current is reduced from $11 \mathrm{~A}$ to $5.5 \mathrm{~A}$ while the fundamental frequency is also reduced from $50 \mathrm{~Hz}$ to $25 \mathrm{~Hz}$. This case is particularly relevant for drives applications. When this change is applied, the SHE-PWM pattern goes from $N_{\alpha}=5$ to $N_{\alpha}=7$ angles. In Fig. 10(f) one can observe that the proposed predictive controller leads the current to its new reference in about $5 \mathrm{~ms}$. This clearly shows that the proposed SHE-MPC strategy can easily handle multiple SHEPWM patterns.

\section{CONCLUSION}

In this work, a predictive control formulation for power converters that achieves closed-loop when using SHE-PWM has been proposed. As evidenced by the results, the proposed SHE-MPC strategy exhibits a fast closed-loop dynamic. To achieve this, the predictive controller slightly modifies the optimal SHE-PWM pattern during transients. Regarding the steady-state, the proposed SHE-MPC is able to resemble the optimized voltage pattern without introducing undesired commutations or oscillations. Consequently, the targeted low order harmonics are effectively canceled. This is a major advantage of the proposed SHE-MPC when compared to converters governed by conventional PI controllers. Moreover, this is accomplished using only a one-step prediction which is a major contribution since, until now, only long-horizon predictive control formulations have been shown to have the potential to achieve a performance similar to that of optimized voltage patterns [27]. In this work, the proposed predictive controller has been successfully combined and tested with an SHE-PWM pattern. However, it can be easily extended to be used with other optimized patterns, such as OPP and SHM.

Future work will be focused on the robustness of the proposed predictive control strategy. Additionally, based on the results presented in this work, novel predictive control strategies with optimized pulse pattern can be developed for more complex cases such as grid-connected converters and drives.

\section{REFERENCES}

[1] S. Kouro, M. Malinowski, K. Gopakumar, J. Pou, L. Franquelo, B. Wu, J. Rodríguez, M. A. Pérez, and J. Leon, "Recent Advances and Industrial Applications of Multilevel Converters," IEEE Transactions on Industrial Electronics, vol. 57, no. 8, pp. 2553-2580, 2010.

[2] H. Abu-Rub, J. Holtz, J. Rodríguez, and G. Baoming, "Medium-Voltage Multilevel Converters-State of the Art, Challenges, and Requirements in Industrial Applications," IEEE Transactions on Industrial Electronics, vol. 57, no. 8, pp. 2581-2596, 2010.

[3] A. Edpuganti and A. K. Rathore, "A Survey of Low Switching Frequency Modulation Techniques for Medium-Voltage Multilevel Converters," IEEE Transactions on Industry Applications, vol. 51, no. 5, pp. 4212-4228, 2015.

[4] J. Leon, S. Kouro, L. Franquelo, J. Rodríguez, and B. Wu, "The Essential Role and the Continuous Evolution of Modulation Techniques for Voltage Source Inverters in Past, Present and Future Power Electronics," IEEE Transactions on Industrial Electronics, vol. 63, pp. 2688-2701, May 2016. 
[5] A. Ghias, J. Pou, G. J. Capella, V. G. Agelidis, R. P. Aguilera, and T. A. Meynard, "Single-Carrier Phase-Disposition PWM Implementation for Multilevel Flying Capacitor Converters," IEEE Transactions on Power Electronics, vol. 30, pp. 5376-5380, Oct. 2015.

[6] H. S. Patel and R. G. Hoft, "Generalized Techniques of Harmonic Elimination and Voltage Control in Thyristor Inverters: Part I-Harmonic Elimination," IEEE Transactions on Industry Applications, no. 3, pp. 310-317, 1973 .

[7] M. S. A. Dahidah, G. Konstantinou, and V. G. Agelidis, "A Review of Multilevel Selective Harmonic Elimination PWM: Formulations, Solving Algorithms, Implementation and Applications," IEEE Transactions on Power Electronics, vol. 30, no. 8, pp. 4091-4106, 2015.

[8] J. Holtz and N. Oikonomou, "Synchronous Optimal Pulsewidth Modulation and Stator Flux Trajectory Control for Medium-Voltage Drives," IEEE Transactions on Industry Applications, vol. 43, no. 2, pp. 600-608, 2007.

[9] T. Geyer, "A Comparison of Control and Modulation Schemes for Medium-Voltage Drives: Emerging Predictive Control Concepts Versus PWM-Based Schemes," IEEE Transactions on Industry Applications, vol. 47, no. 3, pp. 1380-1389, 2011.

[10] P. Cortés, M. P. Kazmierkowski, R. M. Kennel, D. E. Quevedo, and J. Rodríguez, "Predictive Control in Power Electronics and Drives," IEEE Transactions on Industrial Electronics, vol. 55, no. 12, pp. 4312 4324, Dec. 2008.

[11] S. Kouro, P. Cortés, R. Vargas, U. Ammann, and J. Rodríguez, "Model Predictive Control-A Simple and Powerful Method to Control Power Converters," IEEE Transactions on Industrial Electronics, vol. 56, no. 6, pp. 1826-1838, 2009

[12] J. Rodríguez, M. P. Kazmierkowski, J. Espinoza, P. Zanchetta, H. AbuRub, H. A. Young, and C. A. Rojas, "State of the Art of Finite Control Set Model Predictive Control in Power Electronics," IEEE Transactions on Industrial Informatics, vol. 9, no. 2, pp. 1003-1016, May 2013.

[13] S. Vazquez, J. Leon, L. Franquelo, J. Rodríguez, H. A. Young, A. Marquez, and P. Zanchetta, "Model Predictive Control: A Review of Its Applications in Power Electronics," IEEE Industrial Electronics Magazine, vol. 8, no. 1, pp. 16-31, 2014.

[14] D. E. Quevedo, R. P. Aguilera, and T. Geyer, "Predictive Control in Power Electronics and Drives: Basic Concepts, Theory, and Methods," in Studies in Computational Intelligence. New York, NY, USA: Springer, 2014, pp. 181-226.

[15] R. P. Aguilera, P. Lezana, and D. E. Quevedo, "Finite-Control-Set Model Predictive Control With Improved Steady-State Performance," IEEE Transactions on Industrial Informatics, vol. 9, no. 2, pp. 658-667, May 2013.

[16] P. Cortés, J. Rodríguez, D. E. Quevedo, and C. Silva, "Predictive Current Control Strategy With Imposed Load Current Spectrum," IEEE Transactions on Power Electronics, vol. 23, no. 2, pp. 612-618, 2008.

[17] R. P. Aguilera, P. Lezana, and D. E. Quevedo, "Switched Model Predictive Control for Improved Transient and Steady-State Performance," IEEE Transactions on Industrial Informatics, vol. 11, no. 4, pp. 968977, Aug. 2015

[18] S. Kouro, B. La Rocca, P. Cortés, S. Alepuz, B. Wu, and J. Rodríguez, "Predictive control based selective harmonic elimination with low switching frequency for multilevel converters," in 2009 IEEE Energy Conversion Congress and Exposition, San Jose, CA, Sep. 2009, pp. 3130-3136.

[19] R. P. Aguilera, P. Lezana, G. Konstantinou, P. Acuna, B. Wu, S. Bernet, and V. G. Agelidis, "Closed-loop SHE-PWM technique for power converters through Model Predictive Control," in Industrial Electronics Society, IECON 2015 - 41st Annual Conference of the IEEE, Yokohama, Japan, Nov. 2015, pp. 005 261-005 266.

[20] V. G. Agelidis, A. I. Balouktsis, and C. Cossar, "On Attaining the Multiple Solutions of Selective Harmonic Elimination PWM ThreeLevel Waveforms Through Function Minimization," IEEE Transactions on Industrial Electronics, vol. 55, no. 3, pp. 996-1004, 2008.

[21] J. Napoles, J. Leon, R. Portillo, L. Franquelo, and M. A. Aguirre, "Selective Harmonic Mitigation Technique for High-Power Converters," IEEE Transactions on Industrial Electronics, vol. 57, no. 7, pp. 23152323,2010

[22] D. E. Quevedo, R. P. Aguilera, M. A. Pérez, P. Cortés, and R. Lizana, "Model Predictive Control of an AFE Rectifier With Dynamic References," IEEE Transactions on Power Electronics, vol. 27, no. 7, pp. 3128-3136, 2012.

[23] V. Yaramasu, B. Wu, and J. Chen, "Model-Predictive Control of GridTied Four-Level Diode-Clamped Inverters for High-Power Wind Energy Conversion Systems," IEEE Transactions on Power Electronics, vol. 29, no. 6, pp. 2861-2873, 2014.
[24] V. Yaramasu, M. Rivera, B. Wu, and J. Rodríguez, "Model Predictive Current Control of Two-Level Four-Leg Inverters-Part I: Concept, Algorithm, and Simulation Analysis," IEEE Transactions on Power Electronics, vol. 28, no. 7, pp. 3459-3468, Jul. 2013.

[25] V. Yaramasu and B. Wu, "Predictive Control of a Three-Level Boost Converter and an NPC Inverter for High-Power PMSG-Based Medium Voltage Wind Energy Conversion Systems," IEEE Transactions on Power Electronics, vol. 29, no. 10, pp. 5308-5322, 2014.

[26] T. Geyer and D. E. Quevedo, "Multistep Finite Control Set Model Predictive Control for Power Electronics," IEEE Transactions on Power Electronics, vol. 29, no. 12, pp. 6836-6846, 2014.

[27] — , "Performance of Multistep Finite Control Set Model Predictive Control for Power Electronics," IEEE Transactions on Power Electronics, vol. 30, no. 3, pp. 1633-1644, Mar. 2015.

[28] P. Acuna, L. Moran, M. Rivera, R. P. Aguilera, R. Burgos, and V. G Agelidis, "A Single-Objective Predictive Control Method for a Multivariable Single-Phase Three-Level NPC Converter-Based Active Power Filter," IEEE Transactions on Industrial Electronics, vol. 62, no. 7, pp. 4598-4607, Jul. 2015.

[29] S. Vazquez, A. Marquez, R. Aguilera, D. Quevedo, J. Leon, and L. Franquelo, "Predictive Optimal Switching Sequence Direct Power Control for Grid-Connected Power Converters," IEEE Transactions on Industrial Electronics, vol. 62, no. 4, pp. 2010-2020, Apr. 2015.

[30] W. Xie, X. Wang, F. Wang, W. Xu, R. Kennel, and D. Gerling, "Dynamic Loss Minimization of Finite Control Set-Model Predictive Torque Control for Electric Drive System," IEEE Transactions on Power Electronics, vol. 31, no. 1, pp. 849-860, Jan. 2016.

[31] Z. Zhang, F. Wang, T. Sun, J. Rodríguez, and R. Kennel, "FPGA-Based Experimental Investigation of a Quasi-Centralized Model Predictive Control for Back-to-Back Converters," IEEE Transactions on Power Electronics, vol. 31, no. 1, pp. 662-674, Jan. 2016.

[32] R. Vargas, U. Ammann, and J. Rodríguez, "Predictive Approach to Increase Efficiency and Reduce Switching Losses on Matrix Converters," IEEE Transactions on Power Electronics, vol. 24, no. 4, pp. 894-902, 2009.

[33] R. P. Aguilera and D. E. Quevedo, "Predictive Control of Power Converters: Designs With Guaranteed Performance," IEEE Transactions on Industrial Informatics, vol. 11, no. 1, pp. 53-63, 2015.

[34] — , "On stability and performance of finite control set MPC for power converters," in Predictive Control of Electrical Drives and Power Electronics (PRECEDE), 2011 Workshop on, Munich, Germany, Oct. 2011, pp. 55-62.

[35] — "Stability Analysis of Quadratic MPC with a Discrete Input Alphabet," IEEE Transactions on Automatic Control, vol. 58, no. 12, pp. 3190-3196, Dec. 2013.

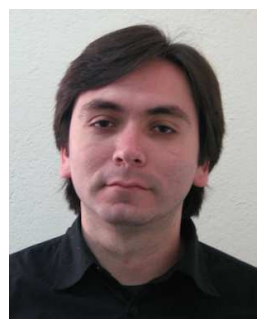

Ricardo P. Aguilera (S'01-M'12) received his B.Sc. degree in Electrical Engineering from the Universidad de Antofagasta, Chile, 2003, M.Sc. degree in Electronics Engineering from the Universidad Técnica Federico Santa María (UTFSM), Valparaíso, Chile, 2007, and Ph.D. degree in Electrical Engineering from The University of Newcastle, Australia, 2012. From 2003 to 2004, he was a Research Assistant at the UTFSM. Then, from 2012 to 2013, he was a Research Academic at the School of Electrical Engineering and Computer Science at The University of Newcastle, Australia, where he was part of the Centre for Complex Dynamic Systems and Control.

In January 2013, he joined the School of Electrical Engineering and Telecommunications at The University of New South Wales (UNSW), Sydney, Australia, where he currently holds a Senior Research Associate position at the Australian Energy Research Institute (AERI). His main research interests include power electronics, renewable energy, and theoretical and practical aspects on model predictive control. 


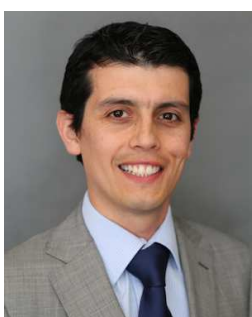

Pablo Acuña (M'12) received the B.S. in Electronics Engineering, the Electronics Engineering Professional degree, and the Ph.D. degree in Electrical Engineering from the University of Concepción, Chile, in 2004, 2007, and 2013 respectively. He is currently Research Associate at the Australian Energy Research Institute (AERI), School of Electrical Engineering and Telecommunications, University of New South Wales, Sydney, Australia. His main research interests include the areas of power modeling and applications. electronics, power quality, and electric power system

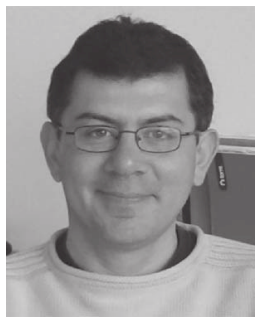

Pablo Lezana (S'06-M'07) was born in Temuco, Chile, in 1977. He received the M.Sc. and Doctor degrees in electronic engineering from the Universidad Técnica Federico Santa María (UTFSM), Valparaíso, Chile, in 2005 and 2006, respectively.

From 2005 to 2006, he was a Research Assistant with the Departamento de Electrónica, UTFSM. From 2007 to 2009, he worked as Researcher at the Departamento de Ingeniería Eléctrica, UTFSM, and since 2010 he holds an Associate Professor position at the same department. Since January 2013 he is the Head of the Departamento de Ingeniería Eléctrica, UTFSM. Since 2015 he is Vice Director of the Advanced Center for Electrical and Electronic Engineering (AC3E) Basal Center, funded by the Chilean Research Council (CONICYT).

His research interests include topologies and control of power converters and modern digital control devices (DSPs and Field Programmable Gate Arrays). Dr. Lezana received the IEEE TRANS. ON INDUSTRIAL ELECTRONICS Best Paper Award in 2007.

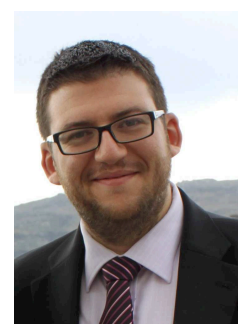

Georgios Konstantinou (S'08-M'11) received the B.Eng. degree in electrical and computer engineering from the Aristotle University of Thessaloniki, Thessaloniki, Greece, in 2007 and the Ph.D. degree in electrical engineering from UNSW Australia, Sydney, in 2012. He is currently a Lecturer with the School of Electrical Engineering and Telecommunications at UNSW Australia. His research interests include multilevel power electronics converters and their applications, pulse width modulation and selective harmonic elimination techniques.

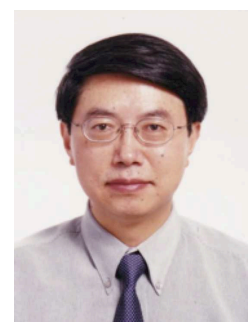

Bin Wu (S'89-M'92-SM'99-F'08) received his M.A.Sc. and Ph.D. degrees in electrical and computer engineering from the University of Toronto, Canada in 1989 and 1993, respectively. He joined Ryerson University in 1993, where he is currently a Professor and Senior NSERC/Rockwell Automation Industrial Research Chair in Power Electronics and Electric Drives. Dr. Wu has published more than 350 technical papers, authored/coauthored two Wiley-IEEE Press books, and holds more than 30 granted/pending US/European patents in the area of power conversion, medium voltage drives, and renewable energy systems.

Dr. Wu received the Gold Medal of the Governor General of Canada in 1993, Premiers Research Excellence Award in 2001, NSERC Synergy Award for Innovation in 2002, Ryerson Distinguished Scholar Award in 2003, Ryerson YSGS Outstanding Contribution to Graduate Education Award and Professional Engineers Ontario (PEO) Engineering Excellence Medal in 2014. He is a fellow of Engineering Institute of Canada (EIC) and Canadian Academy of Engineering (CAE).

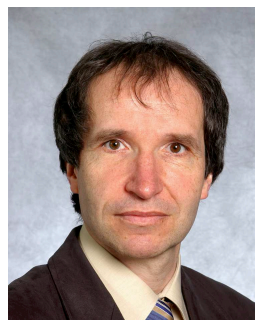

Steffen Bernet (M97) received the diploma degree form Dresden University of Technology in 1990 and the Ph.D. degree from Ilmenau University of Technology in 1995, both in electrical engineering. During 1995 and 1996, he worked as Postdoc in the ECE Department of the University of Wisconsin - Madison. In 1996, he joined ABB Corporate Research, Heidelberg (Germany) where he led the Electrical Drive Systems Group. From 1999 to 2000 he was subprogram manager responsible for the $\mathrm{ABB}$ research in the areas Power Electronics "Systems", "Drives" and "Electric Machines". From 2001 to 2007 he was Professor for Power Electronics at Berlin University of Technology. Since June 2007 he has been Professor at Dresden University of Technology.

During the past twenty years, Dr. Bernet has conducted comprehensive research on power semiconductors, static power converters and ac motor drives. He has received several awards including seven IEEE paper awards.

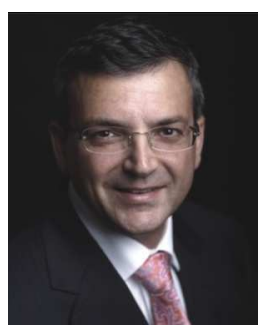

Vasssilios G. Agelidis (SM'00-F'16) was born in Serres, Greece. He received the B.Eng. degree in electrical engineering from the Democritus University of Thrace, Thrace, Greece, in 1988; the M.S. degree in applied science from Concordia University, Montreal, QC, Canada, in 1992; and the Ph.D. degree in electrical engineering from Curtin University, Perth, Australia, in 1997.

He was with Curtin University (1993-1999); the University of Glasgow, Glasgow, U.K. (2000-2004); Murdoch University, Perth, Australia (2005-2006); and the University of Sydney, Sydney, Australia (2007-2010). He is currently the Director of the Australian Energy Research Institute, School of Electrical Engineering and Telecommunications, University of New South Wales (UNSW), Sydney, Australia.

Prof. Agelidis received the Advanced Research Fellowship from the U.K.s Engineering and Physical Sciences Research Council in 2004. He was the Vice President for Operations with the IEEE Power Electronics Society (PELS) from 2006 to 2007. He was an AdCom Member of the IEEE PELS from 2007 to 2009 and the Technical Chair of the 39th IEEE Power Electronics Specialists Conference in 2008 held in Rhodes, Greece. 